\title{
Aktuelle Lehrwerke für den DaF-Unterricht unter dem Aspekt der Phraseodidaktik
}

\author{
SAŠ̀ JAZBEC AND Milka EnČEVA \\ Universität Maribor, Slowenien
}

Received: 18 January $2011 /$ Accepted: 12 June 2011

ISSN: $1697-7467$

ZUSAMMENFASSUNG: Die wissenschaftlichen und fachlichen Erkenntnisse in den letzten Jahrzehnten bezeugen einen Aufschwung in der Phraseologieforschung sowie Phraseodidaktik. Aufgrund dieser Feststellung könnte man u. a. erwarten, dass die aktuellen Lehrwerke diese Erkenntnisse bereits widerspiegeln. Dieser Beitrag hat das Ziel, das Quantum und die Frequenz von Phrasemen sowie ihren Stellenwert in den für den DaF-Unterricht aktuellen Lehrwerken für das Niveau B2 der großen deutschsprachigen Verlage Langenscheidt, Hueber und Klett, die weltweit für den DaFUnterricht verwendet werden, zu analysieren. Die Ergebnisse werden diskutiert und relevante didaktische Richtlinien für ein didaktisches Optimum an Phrasemen für den DaF-Unterricht formuliert.

Schlagworte: Phraseodidaktik, Lehrwerke, Phrasem, phraseologisches Optimum, Geläufigkeit, Frequenz

Current Textbooks for Teaching German as a Foreign Language from the Point of View of Phraseodidactics

\begin{abstract}
Scientific and technical findings in recent decades have witnessed an upturn in phraseology research as well as in phraseodidactics. It could therefore be expected that topical school textbooks would already reflect these findings. This contribution analyzes the number and the frequency of idioms as well as their value in the current school textbooks for teaching German as a foreign language at the B2 level. These have been developed by the big, German-speaking publishing companies, Langenscheidt, Hueber and Klett, and are used worldwide for teaching German as a foreign language. The findings are discussed and relevant didactic guidelines formulated for an optimal pedagogical approach to idioms in teaching German as a foreign language.
\end{abstract}

Keywords: Phraseodidactic, textbooks, idioms, phraseological optimum, fluency, frequency 


\section{EINFÜHRUNG ${ }^{1}$}

Phraseologie als ein Teilbereich linguistischer Forschung erlebte erst in den letzten Jahren - wovon etliche wissenschaftliche und fachliche Publikationen zeugen - zunehmend Konjunktur (Vgl. Fleischer, 1997; Földes, 2004; Burger u. a. 2007; Burger, 2010). Es hat sich gezeigt, dass sie ein weites, forschungsbedürftiges und höchst aktuelles Forschungsfeld ist. Sie prägte und prägt weiterhin nicht nur Linguistik, sondern auch Soziolinguistik, Stilistik, Lexikographie sowie Didaktik und Fremdsprachendidaktik usw. (Vgl. bspw. LorenzBourjot, Lüger, 2001; Kühn, 1994; Bergerová, 2007 u. a.). Im Weiteren wird unsere Aufmerksamkeit der letztgenannten Disziplin - der Fremdsprachendidaktik - im Zusammenhang mit der Phraseologie gewidmet.

Der vorliegende Beitrag grenzt das breite Feld der sog. Phraseodidaktik ${ }^{2}$ ein, indem er sich auf die folgenden Fragestellungen konzentriert: Wie ist es um das Quantum und die Frequenz von Phrasemen bestellt sowie welchen Stellenwert haben sie in den für den DaF-Unterricht aktuellen Lehrwerken für das Niveau B2 der großen deutschsprachigen Verlage Langenscheidt, Hueber und Klett, die weltweit für den DaF-Unterricht verwendet werden? Anders formuliert: Enthalten die Lehrwerke das phraseologische Minimum, Optimum oder sogar Maximum?

In den bereits existierenden Untersuchungen zum Thema Fremdsprachenunterricht und Phraseme - erforscht wurden Lernerwörterbücher und andere Nachschlagewerke für Deutsch als Fremdsprache (Vgl. Wotjak, 2001; Hallsteinsdóttir et. al., 2006) - wurde die sog. ,phraseologische Enthaltsamkeit‘ (Beyer, 2003: 72) festgestellt bzw. es zeigte sich, dass das Maß an Phrasemen (für Parömien kann das nicht behauptet werden) zwar adäquat sei, jedoch didaktisch nicht ausreichend genutzt werde (Vgl. ebenda). ${ }^{3}$ Wenn man aber vom oben erwähnten Aufschwung in der Phraseologieforschung ausgeht, den die wissenschaftlichen und fachlichen Erkenntnisse in den letzten Jahrzehnten bezeugen, könnte man daraus schlussfolgern, dass diese auch in den aktuellen Lehrwerken bereits reflektiert werden. Die in den Lehrwerken enthaltenen Phraseme werden auf die oben genannten Fragestellungen hin analysiert und dann relevante didaktische Implikationen und Richtlinien für ein didaktisches Optimum an Phrasemen für den DaF-Unterricht formuliert.

${ }^{1}$ Der Beitrag ist im Rahmen des Forschungsprojektes mit dem Titel: Phraseologie der deutschen Sprache. Deutsch-slowenische interkulturelle und kontrastive Aspekte (J6-3601) entstanden. Das Projekt dauert von 2010 bis 2013 und wird von der slowenischen Agentur AARS finanziell unterstützt.

${ }^{2}$ Derjenige Teil der Didaktik, der sich mit der Phraseologie im Kontext der Fremdspachendidaktik, mit Vermittlung von Phraseologismen im Unterricht auseinandersetzt, wird Phraseodidaktik genannt.

${ }^{3}$ Die Fremdsprachendidaktik weist zwar der Phraseologie eine wichtige Rolle zu, verweist aber stets auf Mankos und Mängel auf diesem Gebiet im Fremdsprachenunterricht. Diese Mankos und Mängel beziehen sich sowohl auf die Ebene der Lehrwerke und Lehrmaterialien als auch auf die Spontaneität und Unsystematisiertheit vorhandener Phraseologismen darin (Beyer 2003). 


\section{Phraseme in der linguistik - Begriffskussion Und - eingrenZung}

Wenn man in einem bunten Spektrum von Texten, Textsorten, Anweisungen, Aufgabenstellungen u. a. m., die die Lehrwerke enthalten, die erfassten Phraseme und untersuchen will, dann ist es notwendig zunächst relevante theoretische Grundlagen zu präsentieren und somit die Auswahlkriterien für die zu ermittelnden Phraseme zu bestimmen. Wann kann man ein sprachliches Phänomen als ein Phrasem kennzeichnen? Wie unterscheidet man ein Phrasem von einem ,Nicht-Phrasem'? Welche Eigenschaften hat eine sprachliche Einheit, die als Phrasem bezeichnet werden kann? Den weiteren Überlegungen zu den Fragestellungen muss Folgendes vorausgeschickt werden: Angesichts der Breite der Thematik kann der vorliegende Beitrag in der Form keinen Anspruch auf Vollständigkeit erheben, sondern der theoretische Rahmen wird im Lichte einer relevanten und signifikanten Analyse eingegrenzt.

In der aktuellen Phraseologie werden mehrere Definitionen ${ }^{4}$ zur Diskussion gestellt (Vgl. z. B. Donalies, 2009; Burger, 2010). Es gibt im Wesentlichen drei Kriterien (ein morphologisches, ein syntaktisches und ein semantisches) oder nach Ehrhard „Definitionsstränge» (1999: 39), nach denen der Hauptgegenstand der Phraseologie definiert und von anderen abgegrenzt wird. Infolge der unterschiedlichen Berücksichtigung dieser Kriterien kam es zu einer ,terminologischen Unübersichtlichkeit» (Stein, 1995: 45) oder „verwirrenden Terminologie» (Donalies, 2009: 29). So setzt Donalies die primäre Berücksichtigung eines Kriteriums in Beziehung zu der jeweiligen Bezeichnung des Gegenstandes der Phraseologie, was aus der folgenden Tabelle ersichtlich wird.

\begin{tabular}{|l|l|}
\hline & $\begin{array}{l}\text { Verschiedene Bezeichnungen für die Grundeinheit der } \\
\text { Phraseologie }\end{array}$ \\
\hline Polylexikalität & $\begin{array}{l}\text { Phrasem, Phraseologismus, syntaktische Gruppe, Syntagma, } \\
\text { Wortgruppe, Wortfügung, Wortverknüpfung }\end{array}$ \\
\hline $\begin{array}{l}\text { Syntaktische Anomalien } \\
\text { und Fixiertheit }\end{array}$ & $\begin{array}{l}\text { feste Wortgruppe, feste Verbindung, festes Syntagma } \\
\text { festgeprägter Satz, fixiertes Wortgefüge, stehende } \\
\text { Redewendung, Fertigbauteile, autonomes Syntagma. }\end{array}$ \\
\hline $\begin{array}{l}\text { Wiederholung bzw. } \\
\text { Frequenz }\end{array}$ & $\begin{array}{l}\text { Kollokation, Kookurrenz, Redensart, Gemeinplatz, Formel, } \\
\text { Floskel, Stereotyp, Klischee, automatisierter Redeteil }\end{array}$ \\
\hline $\begin{array}{l}\text { Idiomatizität bzwatische } \\
\text { Nichtidiomatizität }\end{array}$ & $\begin{array}{l}\text { Idiom, Idiomatismus, idiomatische Wendung, idiomatische } \\
\text { Phrase, idiomatische Lexemkette }\end{array}$ \\
\hline $\begin{array}{l}\text { Versprachlichung des } \\
\text { Begriffs }\end{array}$ & $\begin{array}{l}\text { Phraseolexeme, Wortgruppenlexeme, phraseologische } \\
\text { Einheit }\end{array}$ \\
\hline
\end{tabular}

(Vgl. Donalies, 2009: 30f.)

\footnotetext{
${ }^{4}$ Die definitorische Festlegung des Gegenstandes der Phraseologie ist sowohl für die Phraseodidaktik im muttersprachlichen Unterricht und im Fremdsprachenunterricht als auch für die Phraseographie bzw. pädagogische Lexikographie und deren Einsatz im Sprachunterricht von großer Bedeutung. Deshalb ist es erstaunlich, dass die linguistische Darstellung der Phraseme erst in den 70er Jahren des vorigen Jahrhunderts aufkam. Das kann einerseits auf die späte Etablierung der Phraseologie als eigenständiger
} 
Trotz der überaus verwirrenden Vielzahl an Termini für den Hauptgegenstand der Phraseologie haben sich in der deutschen Phraseologieforschung zwei auch international häufig gebräuchliche Termini etabliert - Phrasem und Idiom. Der Terminus „Phrasem» hat sich aus zwei Gründen durchgesetzt: Die Bezeichnung legt nahe, dass die Grundeinheit der Phraseologie durch die Polylexikalität und eine syntaktische Struktur gekennzeichnet wird. Außerdem passt sie in eine Reihe international verbreiteter Termini für linguistische Bereiche und ihre Hauptgegenstände wie z. B.

Phonologie - Phonem
Morphologie - Morphem
Lexikologie - Lexem
Phraseologie - Phrasem

(ähnlich auch Donalies, 2009: 31; Burger, 2010: 35)

„Idiom» ist der international gebräuchlichste Terminus. Der Hinweis auf das Merkmal Idiomatizität ist offensichtlich. Das Problem bei der Bezeichnung „Idiom» besteht darin, dass man sie in vielen Fällen als Oberbegriff für idiomatische und nichtidiomatische Verbindungen gebraucht (z. B. Dobrovol'ski, 1995). Durch diese Doppeldeutigkeit verliert der Terminus an Eindeutigkeit und Klarheit. In diesem Beitrag wird der Begriff „Phrasem» als Bezeichnung für den Hauptgegenstand der Phraseologie verwendet (ähnlich Jesenšek, 2006; Burger, Dobrovol'ski, Kühn, Norrick, 2007; Donalies, 2009).

Die Phraseologen müssen sich nicht nur auf den Terminus für die Grundeinheit der Phraseologie einigen, sondern auch auf deren Extension. Darum müssen wir an dieser Stelle auch den Begriff Phrasem präzise definieren, indem wir ihn in seinen wesentlichen Merkmalen erfassen und ihn von ähnlichen Begriffen abgrenzen. Dafür wird das Phrasem nach den bereits angeführten morphologischen, syntaktischen und semantischen Kriterien beschrieben.

\subsection{Polylexikalität}

Seit Ch. Bally (1909) wird auf Polylexikalität als wichtiges Merkmal der Phraseme hingewiesen (nach Donalies, 2009: 3). Nach Burger ist es kein Problem zu entscheiden, ob ein Ausdruck aus mehreren Wörtern besteht, ,insofern man sich darauf einigen kann, was ein Wort ist»(2007: 15). Es geht um die Unterscheidung zwischen Autosemantika (Inhaltswörter) und Synsemantika (Funktionswörter). Daraus ergeben sich verschiedene Auffassungen über die Minimalstruktur des Phrasems - je nachdem, ob die Verbindung aus zwei Synsemantika wie z. B. bis an als Phrasem betrachtet werden kann (Vgl. Gréciano, 1997: 169; Donalies, 2009: 10; Burger, 2010: 36) oder nur die Verbindungen aus einem Synsemantikum und einem Autosemantikum wie z. B. an Bord (Fleischer, 1997: 83) oder aber erst die Verbindungen nur aus Autosemantika wie z. B. schwarzer Markt (Lüger, 1999: 6). Schließlich ist es möglich, polylexikalische Einheiten erst ab der Satzgrenze als Phraseme zu behandeln (Wotjak, 1999: 52).

Disziplin in der deutschen Linguistik zurückgeführt werden, andererseits aber auf eine enge, auf Grammatik konzentrierte Orientierung der Linguistik und auf ein Grammatikkonzept, das die Grammatik vor allem auf die Morphosyntax reduzierte und soauch die Semantik und das Lexikon aus der Grammatik ausschloss (Vgl. Helbig, 2006: 165). 
Wir schließen uns der Auffassung an, ,alle usuellen Verbindungen von mindestens zwei Wörtern - welcher Art auch immer - als Phraseme gelten» zu lassen (Donalies, 2009: 10). Dafür setzt sich auch Jesenšek ein, wenn sie den Begriff Phrasem für die Erstellung der lernorientierten mehrsprachigen phraseologischen Datenbank im Rahmen des EphrasProjekts (2006) definiert. Solche phraseologischen Datenbanken seien polyfunktional und würden zum Zwecke der Erweiterung und/oder Weiterentwicklung der phraseologischen Kompetenz, der Deckung textbezogener Funktion in rezeptiven und produktiven Benutzungssituationen und der problemorientierten Konsultation bei der Übersetzung entwickelt. Darum bevorzugt sie ein breiteres Phraseologie-Verständnis (Vgl. Jesenšek, 2008: 131).

$\mathrm{Da}$ es in diesem Beitrag um Sammlungen von Phrasemen geht, die aus Lehrwerken für den DaF-Unterricht exzerpiert werden und auch polyfunktional sind, ist es u. E. angebracht, so wie die Ausführung oben zeigt, von einem breiteren Phrasembegriff auszugehen.

\subsection{Festigkeit}

Lange Zeit wurde die Reproduzierbarkeit bzw. die Wiederholung der Phraseme in der Kommunikation als das entscheidende Kriterium bei ihrer Definition angesehen (Vgl. Fleischer, 1997: 63). Fleischer und Elspaß dagegen lehnen die Reproduzierbarkeit als Kriterium für die Definition und Abgrenzung der Phraseme ab, weil es bereits in anderen Merkmalen wie Wiederholung, Häufigkeit, Stabilität, Üblichkeit enthalten sei (Vgl. Fleischer, 1997: 67; Elspaß, 1998: 44). Donalies weist darauf hin, „wiederholt, reproduziert ist mehr oder weniger alles in der Sprache» und lehnt ebenfalls Wiederholung und Reproduzierbarkeit als ein zuverlässiges Kriterium für die Definition eines Phrasems ab (Vgl. Donalies, 2009: 12f). Außerdem sind reine Frequenzanalysen auch nicht geeignet, Phraseme von anderen Wortverbindungen abzugrenzen, denn dann würden alle hochfrequenten Verbindungen als Phraseme analysiert (Vgl. Bahns, 1996: 27).

Viele Linguisten halten die syntaktische Anomalie für das wichtigste Unterscheidungsmerkmal der Phraseme im Vergleich zu den freien Wortverbindungen. Die Phraseme seien so fixiert, dass sie sich üblichen syntaktischen Operationen sperrten (Donalies, 2009: 14). Wir verzichten auf eine ausführlichere Beschreibung der von diesen Linguisten unternommenen Transformationen, mit denen man das Fixe, das Stabile zu ermitteln versucht, weil dies den Rahmen dieses Beitrags sprengen würde (Vgl. dazu mehr Lüger, 1999; Valentin, 1999; Chrissou, 2000). Dank der Ergebnisse neuerer Untersuchungen konnte überzeugend bewiesen werden, dass sich Phraseme ,wie normale syntaktische Gebilde verhalten» (Burger, 1998: 17) und dass Phraseme „nicht in der Regel an ihrer äußeren Form erkannt werden [können], denn formal gibt es nur selten einen Unterschied (i. e. morphosyntaktische Abweichungen) zu frei gebildeten Sätzen» (Hallsteinsdóttir, Faro, 2006: o. S.)

Bei der Behandlung der Festigkeit als wichtiges Identifizierungsmerkmal von Phrasemen unterscheidet Burger drei Ebenen der Betrachtung - eine psycholinguistische, eine strukturelle und eine pragmatische:

* Unter psycholinguistischer Festigkeit der Phraseme versteht er die mentale Speicherung der Phraseme als Einheiten, ähnlich wie dies bei den Wörtern der 
Fall ist, und die Möglichkeit sie als Ganzes abzurufen und zu produzieren. Zugleich räumt er aber ein, dass die Betrachtung der Festigkeit unter dem psycholinguistischen Aspekt nicht zur Abgrenzung der Phraseme von den Gegenständen anderer linguistischer Gebiete beitragen kann (Burger, 2010: 19).

* Unter Festigkeit im strukturellen Sinne versteht Burger Festigkeit in Bezug auf das sprachliche System. Er hebt hervor, dass „die strukturelle, insbesondere die lexikalische Art von Festigkeit in Bezug auf weite Bereiche der Phraseologie sehr stark zu relativieren [ist]» (Burger, 2010: 23). Dafür sprechen auch die Ergebnisse der jüngeren Phraseologieforschung, die zeigen, dass die Mehrheit der Phraseme keine absolute Festigkeit aufweist, sondern variabel ist und nicht auf eine vollständig fixierte Grundform zurückzuführen ist. Neben Variation ist auch Modifikation von Phrasemen möglich, wenn sie für die Zwecke eines Textes abgewandelt werden.

* Die pragmatische Festigkeit bezieht sich nur auf eine Klasse von Phrasemen, die in bestimmten Situationstypen oder im Bereich der Gesprächssteuerung eine Funktion haben. Diese als Formeln bezeichneten Phraseme haben eine geringe strukturelle Festigkeit (Vgl. Burger, 2010: 28f).

In Anlehnung an Burger halten wir fest, dass Phraseme polylexikalische Einheiten von relativer Festigkeit sind. Sie bestehen aus stabileren oder weniger stabilen Verbindungen von Elementen, ohne dadurch ihren Status als linguistische oder psycholinguistische Einheiten $\mathrm{zu}$ verlieren.

\subsection{Idiomatizität}

Idiomatizität ist das am häufigsten genannte Kriterium zur Abgrenzung von Phrasemen. Wenn sich die Gesamtbedeutung einer polylexikalischen Einheit nicht aus den Teilbedeutungen der lexikalischen Bestandteile erschließen lässt, spricht man von idiomatischer Bedeutung. Idiomatizität ist eine graduelle Eigenschaft, sodass man vollidiomatische (z. B. jmdm. einen Korb geben), teilidiomatische (z. B. blinder Passagier) und nichtidiomatische (z. B. sich die Zähne putzen) Phraseme unterscheiden kann, je nachdem ob jeder Bestandteil, nur einer oder keiner idiomatisch ist.

Idiomatizität ist jedoch nicht nur das am häufigsten genannte, sondern auch „eines der umstrittensten Kriterien» (Durèo, 1994: 35), weil man sie als ein notwendiges oder als ein mögliches Kriterium betrachten kann. Die ersten Phraseologieforscher hielten Idiomatizität für ein notwendiges Kriterium und schlossen alle nichtidiomatischen polylexikalischen Einheiten aus dem Bereich der Phraseologie aus. Die Phraseologen jüngerer Zeit zählen zu den Phrasemen auch nichtidiomatische feste Wortverbindungen wie z. B. Maßnahmen treffen, stolzer Vater (Vgl. z. B. Stein, 1995: 30; Palm, 1997: 107; Donalies, 2009: 22; Burger, 2010: 31). In diesem Beitrag betrachten wir Idiomatizität so wie Donalies (2009: 22) ,als Kann-Kriterium», als Kriterium, das vorkommen kann, aber nicht vorkommen muss. 
Aktuelle Lehrwerke für den DaF-Unterricht unter...

\subsection{Klassifikation der Phraseme}

Bei der Analyse der in den Lehrwerken enthaltenen Phraseme werden wir uns an die von Burger (2010) vorgenommene Klassifikation halten. In der heutigen Forschung sind sich die Phraseologen über die Klassifikationskriterien der Phraseme weitgehend einig. Sie gehen von syntaktischen, semantischen und pragmatischen Kriterien aus, die sie unterschiedlich präferieren, sodass die einzelnen Klassifikationen unterschiedlich ausfallen.

Die Klassifikation von Burger wurde eingesetzt, weil er versucht, den Forschungsbereich der Phraseologie als Ganzes zu erfassen. Dafür verwendet er als Kriterium die Zeichenfunktion der Phraseme, die sie in der Kommunikation haben (Burger, 2010: 36), und unterscheidet referentielle, strukturelle und kommunikative Phraseme. Die referentiellen Phraseme beziehen sich auf Objekte, Vorgänge und Sachverhalte der Wirklichkeit, wie z. B. das Schwarze Brett, jmdn. übers Ohr hauen, Morgenstund hat Gold im Mund; die strukturellen haben nur die Funktion, grammatische Beziehungen herzustellen, wie z. B. in Bezug auf, sowohl - als auch; die kommunikativen übernehmen bestimmte Aufgaben bei der Herstellung, dem Vollzug und der Beendigung kommunikativer Handlungen, wie z. B. Guten Tag, ich meine (Die angeführten Beispiele sind von Burger, 2010: 36).

Da die strukturellen Phraseme grammatische Relationen herstellen, werden sie in den Lehrwerken als mehrgliedrige Präpositionen und Konnektoren bzw. als grammatische Einheiten behandelt. Aus diesem Grund werden wir sie bei unserer Analyse nicht berücksichtigen.

Die kommunikativen Phraseme gehören zu den Ausdrücken mit hoher „kommunikativer Dringlichkeit», weil sie zur Bewältigung von Standardsituationen bei der Kommunikation, zur Ablaufgestaltung und Gesprächssteuerung und zum Ausdruck von Sprechhandlungen dienen, so Lüger (1997). Als „,notwendiger Bestandteil der kommunikativen Fähigkeit» (Götz, 1976: 56) werden sie in den gängigen Lehrwerken schon in der ersten Lektion eingeführt und eingeübt. Über ihre Aufnahme in die DaF-Lehrwerke und die Bedeutung ihrer aktiven Beherrschung lässt sich nicht streiten und wir werden sie in unsere Analyse nicht einbeziehen.

Die referentiellen Phraseme werden weiter in nominative (satzgliedwertige) und propositionale (satzwertige) Phraseme eingeteilt, je nachdem ob sie Objekte und Vorgänge bezeichnen wie das Schwarze Brett, jmdn. übers Ohr hauen oder Aussagen über Objekte und Vorgänge darstellen (Morgenstund hat Gold im Mund) (Vgl. Burger, 2010: 36f). Innerhalb der nominativen Phraseme hält Burger eine Untergliederung nach dem Grad der Idiomatizität für sinnvoll und unterscheidet Idiome, Teil-Idiome und Kollokationen (nicht bzw. schwach idiomatische Phraseme). Die am stärksten vertretene reguläre Untergruppe der Kollokationen sind die sog. Funktionsverbgefüge, die in „Profile Deutsch» aufgeführt und folglich in allen Lehrwerken für das Niveau B2 bzw. C1 behandelt werden (Vgl. Glaboniat u. a. 2005: 228).

In diesem Beitrag analysieren wir nur zwei Untergruppen der nominativen Phraseme, und zwar die Idiome und die Teil-Idiome.

Außerdem gilt unsere Aufmerksamkeit weiteren drei speziellen Klassen von Phrasemen:

- den Modellbildungen, wie z. B. x um x (Glas um Glas, Stein um Stein), die von der Basisklassifikation von Burger nicht erfasst werden, 
- den Zwillingsformeln, wie z. B. dick und fett (nicht idiomatisch), klipp und klar (teilidiomatisch), gang und gäbe (idiomatisch), die in allen Ausprägungen von Idiomatizität auftreten,

- den komparativen Vergleichen, wie z. B. flink wie ein Wiesel, die je nachdem wie durchsichtig der Vergleich ist, als Kollokationen oder Teil-Idiome auftreten.

(Die angeführten Beispiele sind von Burger, 2010: 44ff.)

Aufgrund der beschriebenen Eigenschaften von Phrasemen kommen wir zur folgenden Definition: Als Phraseme im weiteren Sinne werden polylexikalische Einheiten von relativer Festigkeit bezeichnet. Wenn zu diesen zwei Eigenschaften noch Idiomatizität als Eigenschaft hinzukommt, sprechen wir von Phrasemen im engeren Sinne. In diesem Beitrag werden die vollidiomatischen und teilidiomatischen Phraseme sowie drei spezielle Klassen von Phrasemen ermittelt, die in der von Burger vorgenommenen Basisklassifikation eine Sonderrolle einnehmen.

\section{Phraseme im daf-unterricht}

Bereits vor etwa dreißig Jahren stellte Fleischer fest: Es dürfte außer Frage stehen, dass der Phraseologie für den Fremdsprachenunterricht eine besondere Bedeutung zukomme. Die Befähigung $\mathrm{zu}$ einer auch nur begrenzten Kommunikation in einer Fremdsprache sei ohne eine minimale Beherrschung der Phraseologie nicht möglich (Fleischer, 1982: 32). Einige Jahre später hebt auch Lüger noch einmal hervor, phraseologische Ausdrücke gehörten ebenso zur sprachlichen Wirklichkeit wie etwa Partikeln, Komposita, Metaphern oder bestimmte umgangsspezifische oder gruppenspezifische Ausdrücke. Als solche stellen sie einen unverzichtbaren aber dennoch schwierigen und interessanten Gegenstand des Fremdsprachenunterrichts dar (Lüger, 1997: 76,116).

Man kann tatsächlich nicht bezweifeln, dass feste Wortverbindungen - was eine sehr allgemeine Auffassung von Phrasemen ist - ein unentbehrlicher Bestandteil jeder Sprache sind. Diese ziehen sich wie ein roter Faden durch alle Ebenen der Sprache und verleihen ihr die ihr eigene Emotionalität, Expressivität und Kolorit. Im DaF-Unterricht gerät jedoch diese ,Selbstverständlichkeit‘ von Phrasemen in der Sprache ins Wanken. Hessky stellt beispielsweise fest, im Zusammenhang mit den Phrasemen im DaF-Unterricht könne man zwei entgegengesetzte Auffassungen betrachten. Einerseits seien die Phraseme bestenfalls als ,Rosinen“ zu verstehen, die auf weit fortgeschrittenen Stufen zu vermitteln seien und deren Erwerb ein ,Luxus' sei. Andererseits seien sie ein Gradmesser des sprachkommunikativen Könnens. Je bewanderter ein Fremdsprachenlerner in Phrasemen sei, desto anspruchsvoller könne er sich in der Fremdsprache ausdrücken (Hessky, 1997: 139). Man könnte vermuten, der im didaktischen Paradigma noch immer tief verankerte kommunikativ ausgerichtete DaF-Unterricht mit seiner ausgeprägten Tendenz, die Kommunikationsfähigkeit der Lerner in der deutschen Sprache zu fordern und zu fördern, verfolgt ganz die zweite Auffassung. Die Übungen und Aufgabenstellungen im DaFUnterricht, möglichst beste und objektiv messbare Ergebnisse sowie das vorherrschende Prinzip vom Einfachen zum Schwierigen stellen allerdings die oben beschriebene zweite Auffassung von Phrasemen im DaF-Unterricht in Frage und sprechen eher für die erste 
,Luxusauffassung' von Phrasemen im DaF-Unterricht. Wie oben bereits erwähnt vertritt auch Lüger die Auffassung, dass Phraseme ein unverzichtbarer Bestandteil des Fremdsprachenunterrichts sein sollten, schlägt aber Vorsicht bei deren Gebrauch vor sowie vor allem gut durchdachte und spezifische didaktische Maßnahmen. Der Einsatz von Phrasemen im DaF-Unterricht sei eine harte Nuss und die Entwicklung einer sog. phraseologischen Kompetenz des Fremdsprachenlerners ist ein langwieriger Prozess (Lüger, 1997: 76). Damit plädiert er einerseits für den Einsatz von Phrasemen auf allen, nicht nur auf den höheren Stufen des Fremdsprachenlernens. Für eine erfolgreiche Auseinandersetzung mit Phrasemen entwickelt er eine detaillierte Übungstypologie (Vgl. ebenda: 100-116), die jedoch ohne relativ gut entwickelte fremdsprachliche Kompetenz kaum zu bewältigen scheint. Daraus lässt sich andererseits schlussfolgern, dass Phraseme doch ein Thema für höhere Stufen des Fremdsprachenlernens sind.

Diese entgegengesetzten Thesen erfuhren eine deutliche Konjunktur durch das prägende Dokument für das Fremdsprachenlernen im 21. Jahrhundert, „Der gemeinsame europäische Referenzrahmen» (GER, 2001). ${ }^{5}$ Mit der Kalibrierung der Fremdsprachenkenntnisse, der genaueren Bestimmung der sog. Kann-Beschreibungen und Deskriptoren für verschiedene Niveaus setzte er Grenzen im Prozess des Fremdsprachenlernens und Fremdsprachenkönnens. Somit wurde Sprachverwendung, etwas, was im Prinzip nicht nivellierbar erscheint, nivelliert. Lehrkräfte bekamen so ein konkretes Instrument in die Hand, das ihnen die Unterrichtsplanung und Evaluation der Kenntnisse erleichterte und sie weltweit vergleichbar machte. Folglich wurden Listen von Vokabeln und grammatischen Strukturen sowie Textsorten für einzelne Stufen erstellt, die bspw. die Lehrwerkproduktion genau bestimmten und sie heute noch bestimmen. Das Resultat dieser für den DaF-Unterricht sicher förderlichen Maßnahmen hatte aber auch andere Folgen. Konkret für Phraseme bedeutete das, dass sie explizit als Thema oder als Ziel erst auf dem Niveau $\mathrm{C}^{6}{ }^{6}$ angeführt werden (GER, 2001: 121). Das Referenzniveau ,kompetente Sprachverwendung' (C1 und C2) ist zwar präzisiert, es ist aber durchaus möglich und notwendig, dass auf den beiden niedrigeren Sprachniveaus auch Phraseme vorkommen. Ohne Phraseme gehe ja Entscheidendes oder zumindest Charakteristisches verloren (Vgl. Lüger, 1997).

Der GER geht genauer auch auf die Kompetenzen der Sprachverwendenden, -lernenden auf dem Niveau C1 und C2 ein (2001: 103-130). Die kommunikative Sprachkompetenz wird im GER in verschiedene Kompetenzen unterteilt, darunter linguistische, soziolinguistische und pragmatische. $\mathrm{Zu}$ der linguistischen Kompetenz gehört u. a. auch lexikalische Kompetenz,

\footnotetext{
${ }^{5}$ Darin werden jenes Wissen und jene Fertigkeiten erfasst, mit denen Sprachlernende im öffentlichen, beruflichen und privaten Bereich sprachlich handlungsfähig werden (GER 2001: 3). Dieses allgemeine Ziel, das ohne Weiteres auch die Kenntnis von Phrasemen als einem unverzichtbaren, ,natürlichen“ Bestandteil der Sprache (Vgl. Kap 3) beinhaltet, wird dann im GER weiter differenziert.

${ }^{6}$ Auszug aus dem GER:»C1 Verfügt über gute Kenntnisse idiomatischer und umgangssprachlicher Wendungen und ist sich der jeweiligen Konnotationen bewusst [...]. C2 Kann ein großes Spektrum an idiomatischen und alltagssprachlichen Redewendungen wieder erkennen und dabei Wechsel im Register richtig einschätzen [...]. Kann Filmen folgen, in denen saloppe Umgangssprache oder Gruppensprache und viel idiomatischer Sprachgebrauch vorkommt. Kann die Sprache zu geselligen Zwecken flexibel und effektiv einsetzen und dabei Emotionen ausdrücken, Anspielungen und Scherze machen.«(2001: 121).
} 
der Kenntnisse der ,festen Wendungen“ (Satzformeln, Sprichwörter, idiomatische Wendungen usw.) zugeordnet werden (GER, 2001: 111). Im Rahmen der soziolinguistischen Kompetenz, die zur Bewältigung der sozialen Dimension des Sprachgebrauchs erforderlich wird, werden Redewendungen, Aussprüche, Zitate und sprichwörtliche Redensarten angestrebt (GER, 2001: 120) und diese sind ein wichtiger Bestandteil der Phraseologie.

In der DaF-Didaktik kann man im Zusammenhang mit Phrasemen also schlussfolgernd zwei Tendenzen beobachten: Einerseits die Bewusstheit über unabdingbare ,natürliche“ Präsenz von Phrasemen auf allen Stufen des Sprachenlernens und -lehrens. Andererseits die Problematik ihres Einsatzes, da sie ein komplexer Gegenstand sind, der in verschiedenen Sprachdomänen angesiedelt ist und dessen Gebrauch spezifischen, nicht immer bestimmbaren Restriktionen unterliegt. Unser Anliegen ist es also festzustellen, inwieweit die phraseologische Kompetenz in den Lehrwerken durch die Präsenz von Phrasemen für das DaF-Lernen bereits auf dem Niveau B2 gefördert wird.

\section{KORPUS UND DIE KRITERIEN DER ANALYSE}

Im empirischen Teil des Beitrags werden Lehrwerke für das DaF-Lernen auf dem Niveau B2 auf die Präsenz von Phrasemen hin analysiert. Es wurden drei Lehrwerke Aspekte 2 (Langenscheidt Verlag), em Hauptkurs (Hueber Verlag), Deutsch mit Grips 2 (Klett Verlag) - ausgesucht und detailliert behandelt. Somit kann nicht von einer repräsentativen Lehrwerkauswahl gesprochen werden, repräsentativ sind jedoch die Verlagswahl (die drei größten DaF-Lehrwerkverlage Deutschlands) und die Wahl der aktuellen Lehrwerke für das Niveau B2. Jeder Verlag stellt verschiedene Lehrwerke für verschiedene Zielgruppen (Jugendliche, Erwachsene, verschiedene Berufsgruppen usw.) her, die Zahl der Lehrwerke für eine Zielgruppe auf einem bestimmten Niveau ist aber meist auf ein Lehrwerk begrenzt. Im Vordergrund dieses Beitrags steht die Idee, exemplarisch zu zeigen, wie die nicht ganz einheitlichen theoretischen Überlegungen im Zusammenhang mit dem Gegenstand Phraseologie im DaF-Unterricht in der Praxis bzw. in den aktuellen Lehrwerken umgesetzt werden und wie man ein geeignetes Analyseinstrument entwickeln, konkret anwenden und für andere, breiter angelegte Untersuchungen zur Verfügung stellen kann.

In den genannten Lehr- und Arbeitsbüchern wurden schriftliche und dazugehörige mündliche Texte einer Analyse unterzogen. Während der Arbeit stellte sich heraus, dass die ausschließliche Analyse von schriftlichen Lehrwerktexten ein leicht verstelltes Bild der enthaltenen Phraseme vermittelt. Die Textsorten, die in den schriftlichen Lehrwerktexten vertreten sind, enthalten ein anderes phraseologisches Quantum als etwa dazugehörige mündliche Texte in den Lehr- und Arbeitsbüchern. Insofern schien es notwendig das ganze Angebot an Lernmaterialien zu untersuchen. Uns interessierte die quantitative und qualitative Dimension der in den Lehrwerkmaterialien enthaltenen Phraseme:

- Wie umfangreich ist der Bestand an Phrasemen in den Lehrwerkmaterialien, und zwar gesondert in den schriftlichen und mündlichen Lehrbuchtexten, und in den schriftlichen und mündlichen Texten im Arbeitsbuch?

- Welche Phraseme sind in den Lehrwerken vertreten? Die erfassten Phraseme wurden zunächst mit dem „Duden - Das große Wörterbuch der deutschen Sprache 
in zehn Bänden» (1999) und auch mit einem modernen, veränderbaren onlineWörterbuch bzw. Redensarten-Index ${ }^{7}$ verglichen. Darüber hinaus wurden die Phraseme noch mit dem Kernbereich des vorgeschlagenen phraseologischen Optimums von Hallsteinsdóttir et. al. (2006) verglichen, da die Relevanz von Phrasemen auf dieser Liste nicht nur im Wörterbuch, sondern auch unter den Sprechern bestätigt wurde.

\subsection{Die Ergebnisse und Analyse}

\subsubsection{Die erfassten Phraseme in den DaF-Lehrwerken - quantitativ}

Das Resultat einer genaueren Lektüre der ausgewählten Lehrwerke auf die Phraseme hin ist zunächst eine Übersichtstabelle mit Zahlen, die die Quantität der Phraseme darin veranschaulichen.

Tabelle 1. Das Quantum an Phrasemen in den ausgewählten Lehrwerken.

\begin{tabular}{|c|c|c|c|c|c|}
\hline Phraseme & Lehrbuch & Arbeitsbuch & $\begin{array}{l}\text { Transkripte } \\
\text { von } \\
\text { Hörtexten }\end{array}$ & $\begin{array}{l}\text { Seitenumfang } \\
\text { des } \\
\text { Lehrwerkes }\end{array}$ & $\begin{array}{l}\text { Seitenumfang } \\
\text { des } \\
\text { Arbeitsbuches }\end{array}$ \\
\hline $\begin{array}{c}\text { Aspekte 2 } \\
\text { Langenscheidt } \\
\text { Verlag }\end{array}$ & $\mathbf{3 8}$ & $\mathbf{6 0}$ & $\mathbf{1 5}$ & $\mathbf{1 9 9}$ & $\mathbf{1 8 4}$ \\
\hline $\begin{array}{c}\text { Deutsch mit } \\
\text { Grips 2 } \\
\text { Klett Verlag }\end{array}$ & $\mathbf{4 6}$ & $\mathbf{6 4}$ & $\mathbf{6}$ & $\mathbf{1 3 6}$ & $\mathbf{1 3 6}$ \\
\hline $\begin{array}{c}\text { em } \\
\text { Hueber Verlag }\end{array}$ & $\mathbf{5 7}$ & $\mathbf{2 2}$ & $\mathbf{1 1}$ & $\mathbf{1 6 0}$ & $\mathbf{1 6 8}$ \\
\hline
\end{tabular}

Aus der Tabelle ist ersichtlich, dass die Zahl der Phraseme in den untersuchten Lehrwerken hinsichtlich ihres Umfangs relativ gering ist: bspw. beim Lehrwerk Aspekte 2 auf jeder fünften Seite ein Phrasem und bei den Lehrwerken Deutsch mit Grips und em auf jeder zweiten Seite. Ferner zeigen die Daten, dass bei allen von uns untersuchten Lehrwerken die Mehrheit der Phraseme, die darin vorkommen, im Prinzip in schriftlichen Texten und nicht so wie bei Matulina, Èolariæ (2007) in den mündlichen Texten, d. h. Transkripten zu vermerken war. ${ }^{9}$ Dieses Ergebnis steht im Widerspruch zu der Feststellung der Fachleute: „Phraseologismen [treten] am dichtesten in gesprochener

\footnotetext{
7 Redensarten-Index erreichbar im Internet unter http://www.redensarten-index.de/ suche.php(5.1.2011)

${ }^{8}$ Der Seitenumfang umfasst alle Seiten des Buches, samt Quellenverzeichniss, Übersichtstabellen für die Grammatik usw.

${ }^{9}$ Dass es auch anders sein kann, zeigt die Untersuchung von Matulina und Èolariæ (2007). Ihre Analyse der regionalen, d. h. kroatischen und bosnischen Lehrwerke für den DaF-Unterricht (in unsere Analyse wurden nur Lehrwerke, die in Deutschland entstehen, einbezogen) hat ergeben, dass es darin
} 
Alltagskommunikation auf[...]»(Burger, Buhofer, Sialm,1982: 144). Da drängt sich die Frage auf, warum diese fast 30 Jahre alte Erkenntnis in deutschen Lehrwerken bisher nicht berücksichtigt wurde.

Es ist auch interessant die erfassten Phraseme in den Arbeitsbüchern zu betrachten. Die Zahl von Phrasemen in den zwei Arbeitsbüchern - Aspekte 2 und Deutsch mit Grips - ist wesentlich höher als in den dazugehörigen Lehrbüchern. Diese Feststellung relativiert das Lehrwerk em mit fast doppelt so vielen Phrasemen im Lehrbuch wie im Arbeitsbuch dann gründlich. Somit muss der Versuch einer allgemeinen Feststellung, die Lehrbücher enthalten weniger Phraseme als die Arbeitsbücher, auf eine Hypothese reduziert werden, die an weiteren Lehrwerken zu testen wäre.

Jedes Phrasem wurde auch auf seine Vorkommenshäufigkeit hin untersucht. Die Analyse zeigt ein überraschendes Ergebnis: Es gibt nur zwei Phraseme, die mehr als einmal vorkommen. Das Phrasem ,eine/keine (große/kleine) Rolle spielen ' wurde in allen Lehrwerken sechsmal ermittelt und das Phrasem „zu Wort kommen» zweimal in einem Lehrwerk. Alle anderen aufgelisteten Phraseme aus den schriftlichen und dazugehörigen mündlichen Lehrwerktexten konnten ohne Ausnahme nur einmal ermittelt werden. Die Auswahlkriterien für die in den Lehrwerken vorkommenden Phraseme sind nicht transparent. Die Tatsache, dass sie nur einmal erscheinen, ist ein Beweis dafür, dass bei der Behandlung der Phraseme keine Kontinuität besteht. Didaktisch gesehen ist es sinnvoll und empfehlenswert, dass bereits vorgekommene Phraseme in irgendeiner Form absichtlich wiederaufgenommen werden, um gefestigt zu werden. Andernfalls sind sie wie Wörter zu behandeln, die in Texten der Lehrwerke vorkommen, ohne auf der Liste der erforderlichen bzw. obligatorischen Lexeme für das entsprechende Niveau zu sein, die nicht weiter behandelt werden, die nur für das Verstehen wichtig sind, nicht aber für den produktiven Sprachgebrauch der Lernenden. Es steckt kein Konzept, keine Strategie zur Vermittlung und zum Lernen von Phrasemen dahinter und das macht nachdenklich. Sie werden so wie Partikeln als natürliche sprachliche Umgebung angeboten, ohne als Lernstoff behandelt zu werden. Damit ist der Anforderung Genüge getan, dass in den Texten Phraseme vorkommen, weil sie ein unentbehrlicher Teil der Sprache sind und weil ihre Präsenz auf allen Stufen des Sprachenlernens und -lehrens nötig ist. Die Frage ist, ob nur ihre Präsenz als natürliche sprachliche Umgebung in Texten auf Niveau B2 ausreicht oder ob sie weiter didaktisch behandelt werden sollen, damit sowohl die rezeptive als auch die produktive phraseologische bzw. kommunikative Sprachkompetenz der Lernenden entwickelt wird.

\subsubsection{Die erfassten Phraseme in den DaF-Lehrwerken - qualitativ}

Die quantitative Übersicht bzw. die erfassten Phraseme wurden weiter qualitativ untersucht, um festzustellen, ob das zwar bescheidene Quantum an Phrasemen in den Lehrwerken qualitativ gesehen besser abschneidet und durchschaubarer als der quantitative

\footnotetext{
viel mehr Phraseme gibt. Die Mehrheit der exzerpierten Phraseme in den untersuchten regionalen Texten war jedoch bei mündlichen Texten zu vermerken (Vgl. ebd. 2007: 101). Diese Tatsache steht im Einklang mit dem Verhältnis von mündlichen und schriftlichen Texten in den regionalen Lehrwerken. Darin dominieren die mündlichen Texte, während in den in Deutschland veröffentlichten Lehrwerken die schriftlichen Texte wesentlich stärker vertreten sind.
} 
Teil systematisiert werden kann. Die in den Lehrwerken ermittelten Phraseme wurden zunächst in „Duden - Das große Wörterbuch der deutschen Sprache in zehn Bänden» (1999) nachgeschlagen, um feststellen zu können, ob sie zum festgelegten sprachlichen Standard gehören. Darüber hinaus schien es uns auch wichtig zu überprüfen, ob die erfassten Phraseme in einem aktuellen, nicht festgelegten, flexiblen phraseologischen online-Korpus zu finden sind, wobei wir uns für den Redensarten-Index - Wörterbuch für Redensarten, Redewendungen, idiomatische Ausdrücke und feste Wortverbindungen (http://www.redensarten-index.de/suche.php) entschieden haben.

Das Resultat dieser Analysen war eine vielfältige Liste mit verschiedenartigen Feststellungen. Es hat sich gezeigt, dass es Phraseme gibt, die in den beiden Nachschlagewerken vorkommen, und auch solche, die nur in einem enthalten sind, sowie solche, die in keinem zu finden waren. Nach welchen Kriterien bestimmte Phraseme in die Nachschlagewerke aufgenommen wurden oder auch nicht, oder anders, warum gerade diese Phraseme in den Lehrwerken vorkommen, konnte anhand dieser Analyse nicht festgestellt werden.

In beiden Nachschlagewerken ist das Kriterium Frequenz/Gebräuchlichkeit von Phrasemen nicht berücksichtigt worden. Den Empfehlungen von Fachleuten (Đurèo, 2001; Hallsteinsdottir et al., 2006) folgend, die neben der Präsenz von Phrasemen in den Wörterbüchern auch ihre Frequenz und Gebräuchlichkeit hervorheben und in die Listen aufnehmen, wurden dann die in den Lehrwerken ermittelten Phraseme noch einer Analyse unterzogen. Sie wurden mit dem Kernbereich eines phraseologischen Optimums für Deutsch als Fremdsprache von Hallsteinsdóttir et. al. (2006) verglichen. Dieses Optimum ist das Ergebnis zweier unabhängig voneinander durchgeführter Untersuchungen, die zueinander in Relation gesetzt wurden. Die erste Untersuchung beschäftigt sich mit der Frequenz deutscher Phraseologismen. Das Ausgangsmaterial stellen alle Phraseologismen aus zwei Lernerwörterbüchern für $\mathrm{DaF}^{10}$ und weiteren Übungs- und Wörterbüchern zur Phraseologie des Deutschen ${ }^{11}$ dar, die im Korpus des Wortschatzlexikons Deutscher Wortschatz (www.wortschatz.uni-leipzig.de) auf ihre Frequenz hin untersucht wurden. Um die Nachteile einer Untersuchung zur Frequenz auszugleichen ${ }^{12}$, wurde eine empirische Befragung von Muttersprachlern nach der Bekanntheit deutscher Phraseme durchgeführt. Ziel der zweiten Untersuchung war es, die Geläufigkeit von Phrasemen zu dokumentieren, die von Muttersprachlern aktiv verwendet werden. Das Phraseologiekorpus für die Fragebogenuntersuchung wurde durch den Vergleich von fünf phraseologischen Übungs-

${ }^{10}$ Es geht um die Lernerwörterbücher - Wörterbuch Deutsch als Fremdsprache von Kempcke et al. (2000) de Gruyter Verlag und Langenscheidts Großwörterbuch Deutsch als Fremdsprache von Götz et al. (Hrsg.) (1997) Langenscheidt Verlag.

11 Hessky/Ettinger (1997). Deutsche Redewendungen. Ein Wörter-und Übungsbuch für Fortgeschrittene; Griesbach/Schulz (2000). 1000 deutsche Redensarten. Mit Erklärungen und Anwendungsbeispielen; Dobrovol'skij (1997a). Idiome im mentalen Lexikon. Ziele und Methoden der kognitivbasierten Phraseologieforschung.

${ }_{12}$ Als Nachteile einer Untersuchung zur Frequenz werden die Berücksichtigung eines kleinen Teils der Phraseologie, die manuelle bzw. subjektive Erstellung der Suchformen und deren Rolle als einziges Kriterium bei der Festlegung der Frequenz, der Bezug nur auf die geschriebene Sprache in dem verwendeten Korpus zum deutschen Wortschatz der Universität Leipzig angesehen. 
und Lernwörterbüchern ${ }^{13}$ zusammengestellt und enthält 1179 Phraseme. $\mathrm{Zu} 1112$ von ihnen liegen Belege aus der Frequenzuntersuchung vor, die als Grundlage für die vergleichende Untersuchung von Frequenz und Geläufigkeit dienten. Man teilte die 1112 ermittelten Phraseme in je 3 Gruppen nach dem Grad (hoch, mittel, niedrig) ihrer Frequenz bzw. Geläufigkeit ein. Die 3 Gruppen nach der Frequenz im Korpus (A, C, E) wurden zu den 3 Gruppen (B, D, F) nach der Geläufigkeit bei Muttersprachlern in Relation gesetzt, sodass nach dieser Differenzierung 9 Gruppen entstanden. Die Gruppe AB enthält 143 Phraseme mit hoher Frequenz und hoher Geläufigkeit und stellt nach den Autoren den offenen Kernbereich des phraseologischen Optimums für DaF dar (Vgl. Hallsteinsdottir et al., 2006: $127 \mathrm{ff})$.

Da wir die Gruppe AB, um mit Đurèo zu sprechen, als ,ein Extrakt der Korrelation zwischen Bekanntheit und Häufigkeit» betrachten (2001: 104), haben wir die Phraseme aus dieser Gruppe mit den aus den Lehrwerken für das Niveau B2 exzerpierten Phrasemen verglichen. Die Ergebnisse veranschaulicht die Tabelle unten:

Tabelle 2. Phraseme in den ausgewählten Lehrwerken und der Gruppe AB von Hallsteinsdottir et. al (2006).

\begin{tabular}{|c|c|c|c|}
\hline & Lehrbuch & Arbeitsbuch & Transkripte von Hörtexten \\
\hline $\begin{array}{c}\text { Aspekte 2 } \\
\text { Langenscheidt Verlag }\end{array}$ & 4 & 3 & 1 \\
\hline $\begin{array}{c}\text { Deutsch mit Grips } 2 \\
\text { Klett Verlag }\end{array}$ & 3 & 3 & 1 \\
\hline $\begin{array}{c}\text { Em } \\
\text { Hueber Verlag }\end{array}$ & 6 & 16 & 1 \\
\hline
\end{tabular}

Aus der Tabelle 2 wird ersichtlich, dass die Zahl der in den Lehrwerken enthaltenen Phraseme, die auch in der Gruppe $\mathrm{AB}$ des phraseologischen Optimums für DaF von Hallsteinsdóttir et al. (2006) erfasst wurden, äußerst niedrig ist. Eine Ausnahme stellt das Lehrwerk em dar, das im Arbeitsbuch eine deutlich höhere Zahl an ,relevanten' Phrasemen enthält, als das bei den anderen Lehrwerken der Fall ist. Diese Analyse führt zu der Feststellung, dass die Quantität der in den untersuchten Lehrwerken enthaltenen Phraseme gering ist und dazu noch dem von Fachleuten exzerpierten und vorgeschlagenen phraseologischen Kernbereich nicht zugeordnet werden kann.

Das Querlesen von Listen mit Phrasemen aus den untersuchten Lehrwerken brachte ein weiteres interessantes Resultat. Es gibt unter allen aufgefundenen Phrasemen lediglich ein einziges, das in allen Lehrwerken vorkommt und das ist ,eine/keine (große/kleine)

13 Untersucht wurden folgende Lernwörterbücher: Buscha (1979) Deutsches Übungsbuch. 100 Phraseologismen in Bildern; Wotjak/Richter (1993). Sage und schreibe. Deutsche Phraseologismen in Theorie und Praxis; Griesbach/Gudrun (1993). Mit anderen Worten. Deutsche Idiomatik, Redensarten und Redeweisen; Herzog (1993). Deutsche Redewendungen von A-Z; Hessky/Ettinger (1997). Deutsche Redewendungen. Ein Wörter- und Übungsbuch für Fortgeschrittene; Dobrovol'skij (1997b) Idiome der lebendigen Sprache. Deutsch - Russisches Wörterbuch. 
Rolle spielen: Das Phrasem gehört linguistisch gesehen zur neutralen Stilschicht und wird in fast allen Bereichen verwendet, um die Wichtigkeit einer Person oder Sache hervorzuheben, verfügt über eine große innere Varianz und weist eine reguläre formale Struktur auf (Vgl. dazu mehr Ephras Datenbank, 2006). Es wäre interessant zu untersuchen, warum gerade dieses Phrasem in allen analysierten Lehrwerken vorkommt und z. B. das Phrasem ,jemanden unter Druck setzen' u. a. mit ähnlichen Charakteristika nicht belegt wurden, würde aber den Rahmen dieses Beitrags sprengen.

\section{FAZIT}

DaF-Lehrwerke als ein wichtiges, wenngleich nicht einziges sowie oft umstrittenes Mittel der Lehr- und Lernprozesse, sind stets verschiedenen Analysen und Fachdebatten unterzogen. Die meisten Analysen aus verschiedenen Perspektiven (aus der Sicht der didaktisch-methodischen, pädagogischen Forschung, der konstruktivistischen Theorie, der Universalgrammatiktheorie usw.) summieren sich zu einem Generalangriff auf das Medium Lehrwerk, die wenigsten zeigen auf ihre Entwicklungsperspektiven (Vgl. Funk, 2004). Auch die Auseinandersetzung mit den ausgewählten Lehrwerken aus der Sicht der phraseodidaktischen Forschung hat gezeigt, dass sie bei der Anzahl und Auswahl der Phraseme in der Regel sehr große Defizite aufweisen. Man kann sogar behaupten, die analysierten Lehrwerke sind in Bezug auf die nötige Vielfalt und unverzichtbare Präsenz von Phrasemen in der, natürlichen“ Sprache unterbesetzt. Sie weisen lediglich ein bescheidenes, eingeschränktes Angebot an Phrasemen auf, das weder einer Systematik oder Logik unterliegt, noch die theoretischen Grundlagen der Phraseologieforschung befolgt. Die schriftlichen sowie auch dazugehörigen mündlichen Lehrwerktexte sind in Bezug auf Phraseme auf ein nahezu unbedeutendes Minimum reduziert. Die wenigen Phraseme, die in den untersuchten Lehrwerken aufgefunden werden konnten, scheinen von den Lehrwerkautoren spontan ausgewählt zu sein. Diesbezüglich muss die anfangs gestellte Forschungsfrage nach dem phraseologischen Optimum in den Lehrwerken negativ beantwortet werden. Der erfasste und quantitativ sowie qualitativ analysierte Bestand an Phrasemen in den ausgesuchten Lehrwerken für das B2-Niveau lässt die folgende Schlussfolgerung zu: Es kann auf Niveau B2 nicht von einem phraseologischen Minimum gesprochen werden, geschweige denn von einem Optimum. Der exzerpierte Quantum an Phrasemen ist begrenzt und auch didaktisch nicht ausreichend fundiert. Kurzum, Phraseme haben in den untersuchten Lehrwerken für das Niveau B2 kaum einen Stellenwert.

Die vorliegende Untersuchung ist, wie bereits gesagt, nicht repräsentativ, trotzdem weist sie auf wichtige Tendenzen hin, die allerdings an anderen Niveaus sowie in den Lehrwerken anderer Verlage überprüft werden sollten. Vermutlich würde aber eine umfassendere Untersuchung aus der Sicht der phraseodidaktischen Forschung keine wesentlich anderen Ergebnisse hervorbringen, denn nach Funk hätten sich die meisten Lehrwerke der deutschen Verlage auf den ersten Blick auf eine nie gekannte Weise angeglichen (2004: 41).

Es wäre angebracht, auch das gesamte Zusatzangebot im Internet, das jeder Verlag aus marktwirtschaftlichen Gründen bereitstellt und ständig erneuert, einer Lehrwerkanalyse zu unterziehen. Die Texte in diesem Zusatzangebot sind einerseits aktuell(er) als in den 
Lehrwerken, andererseits unterliegt eine solche Untersuchung den Gesetzen des Mediums Internet, den Gesetzen sowie den Möglichkeiten der Veränderbarkeit, Relevanz, Autorschaft von Zusatzmaterialien u. a. m., die bei der Analyse auch berücksichtigt werden müssten.

Der nächste Schritt, der in dieser Untersuchung nicht berücksichtigt wurde, wäre die Lehrwerke auch aus der Sicht der Parömiologie ${ }^{14}$ zu erforschen (Vgl. dazu Kispal, 1999 u. a.). Parömien als ein Teil der Phraseologieforschung sind, so eine allgemeine Beobachtung bei unserer Analyse, in den Lehrwerken der niedrigen Niveaus noch weniger vertreten als Phraseme.

Aus Sicht der Phraseodidaktik ist es wichtig, aus der Kritik an Lehrwerken sowie aus den oben formulierten Forschungsdesideraten auch einige didaktische Implikationen abzuleiten. Es wäre durchaus sinnvoll und angebracht, wenn in den Lehrwerktexten von Anfang an Phraseme enthalten wären. Die Präsenz von ,relevanten', geläufigen sowie frequenten Phrasemen in den Lehrwerktexten auf der rezeptiven Ebene würde den Lernern eine authentische(re) Sprachverwendung vermitteln und somit eher das Hauptlernziel realisieren - die Förderung der fremdsprachlichen Kompetenz. Ein gutes Beispiel ist das Lehrwerk Tangram (Hueber Verlag), das als Zusatzangebot zum Lehrwerk Tangram $1 \mathrm{~A}$ in jeder Einheit bei den Zusatzübungen im Internet ein Phrasem einführt. Der Quantität an Phrasemen wird somit einerseits wieder nicht Genüge geleistet. Auch die Authentizität der fremdsprachlichen Kommunikation könnte hier wieder diskutiert werden. Andererseits wird mit dem Zusatzangebot gezeigt, dass eine systematischere Integration von Phrasemen in den Lernprozess von Anfang an durchaus möglich ist. Das phraseologische Optimum würde somit zwar noch nicht realisiert, die Lerner würden sich aber mit Lehr- und Lernstrategien auseinandersetzen, die sie zum Phrasemerwerb befähigen.

Das Manko an Phrasemen in den Lehrwerken löst nach einer gründlichen Überlegung eine Reihe von anderen allgemeinen didaktisch-methodologischen Fragen aus, wie etwa nach dem Repräsentationsverhältnis zwischen den mündlichen und schriftlichen Lehrwerktexten, die Frage nach der Authentizität und Adaption, nach dem Schwierigkeitsund Didaktisierungsgrad von Lehrwerktexten, inwieweit Phraseme in den Lehrwerktexten didaktisch aufbereitet werden sollten, auf welchem Niveau (B1, B2, C1) neben der Präsenz auf der rezeptiven Ebene der produktive Gebrauch von Phrasemen gefördert werden sollte, u. v. m.

Trotz der Analyseergebnisse, Fragen, Bedenken und Überlegungen, die im Rahmen dieses Beitrag aufgelistet wurden, lässt sich feststellen, dass der aktuelle Stellenwert von Phrasemen in den untersuchten Lehrwerken so unterrepräsentiert ist, dass den Lernenden diesbezüglich nichts anderes übrig bleibt, als sich die ,Rosinen aus dem Kuchen - sprich Lehrwerk - zu picken'.

14 Parömiologie (griech. Paroimia 'Sprichwort') ist eine kulturwissenschaftlich ausgerichtete Disziplin, die die Sprichwörter untersucht. Umstritten ist, ob Letztere zu den Phrasemen gehören sollen. Nach Burger et al. (2007) sei besser auf die Einteilung in Phraseologie und Parömiologie zu verzichten und den ganzen Bereich als Phraseologie zu bezeichnen. 


\section{LiteratURQUELLEN}

\section{Lehrwerke:}

Aspekte 2, Lehrwerk. Deutsch als Fremdsprache für die Mittelstufe [Niveaustufe B2]. Koithan, U. et al. (2008). 1. Auflage. Berlin, München, Wien, Zürich, New York: Langenscheidt.

Aspekte 2, Arbeitsbuch. Deutsch als Fremdsprache für die Mittelstufe [Niveaustufe B2]. Koithan, U. et al. (2008). Berlin, München, Wien, Zürich, New York: Langenscheidt. 1. Auflage. Aspekte 2, Lehrerhandreichungen. Deutsch als Fremdsprache für die Mittelstufe [Niveaustufe B2]. Koithan, U. et al. (2008). Berlin, München, Wien, Zürich, New York: Langenscheidt. 1. Auflage.

Em Neu Hauptkurs, Lehrwerk: Deutsch als Fremdsprache für die Mittelstufe [Niveaustufe B2]. Perlmann-Balme, M. et al. (2010). Ismaning : Max Hueber Verlag. 2. aktualisierte Auflage. Em Neu Hauptkurs, Arbeitsbuch: Deutsch als Fremdsprache für die Mittelstufe [Niveaustufe B2]. Perlmann-Balme, M. et al. (2010). Ismaning : Max Hueber Verlag. 2. aktualisierte Auflage.

Em Neu Hauptkurs, Lehrerhandreichungen: Deutsch als Fremdsprache für die Mittelstufe [Niveaustufe B2]. Perlmann-Balme, M. et al. (2010). Ismaning : Max Hueber Verlag. 2. aktualisierte Auflage.

Deutsch mit Grips, Lehrwerk: Deutsch als Fremdsprache für die Mittelstufe [Niveaustufe B2]. Szablyár, A. et al. (2006). Stuttgart-Budapest: Klett Verlag. 5. aktualisierte Auflage.

Deutsch mit Grips, Arbeitsbuch: Deutsch als Fremdsprache für die Mittelstufe [Niveaustufe B2].

Szablyár, A. et al. (2006). Stuttgart-Budapest: Klett Verlag. 5. aktualisierte Auflage.

Deutsch mit Grips, Lehrerhandreichungen: Deutsch als Fremdsprache für die Mittelstufe [Niveaustufe B2]. Szablyár, A. et al. (2006). Stuttgart-Budapest: Klett Verlag. 5. aktualisierte Auflage.

\section{Andere Quellen}

Bahns, J. (1996). Kollokationen als lexikografisches Problem. Eine Analyse allgemeiner und spezieller Lernerwörterbücher des Englischen. Tübingen: Niemeyer (Lexikographica 74).

Bergerová, H. (2007). »Überlegungen zur Phraseologismenvermittlung im DaF-Unterricht. Ein Didaktisierungsvorschlag«. In Aussiger Beiträge, 1:163-178.

Beyer, J. (2003). „Feste Wendungen - untrennbarer Bestandteil der Wortschatzarbeit in DaFUnterricht». In J. Korèáková and J. Beyer (Hrsg.). Königgrätzer Linguistik- und Literaturtage: Hradec: Universitätsverlag, 68-84.

Burger, H., Buhofer and Sialm, A. (1982). Handbuch der Phraseologie. Berlin: de Gruyter.

Burger, H. (2010). Phraseologie. Eine Einführung am Beispiel des Deutschen. Berlin: Erich Schmidt Verlag. 4. Auflage.

Burger, H., Dobrovol'ski, D., Kühn, P. and Norrick, N. (Hrsg.) (2007). Phraseologie. Ein internationales Handbuch zeitgenössischer Forschung/An International Handbook of Contemporary Research. Berlin, New York: De Gruyter.

Buscha, J.(1979). Deutsches Übungsbuch. 100 Phraseologismen in Bildern. Leipzig: VEB Verlag Enzyklopädie.

Chrissou, M. (2000). Kontrastive Untersuchungen zu deutschen und neugriechischen Phraseologismen mit animalistischer Lexik. Essen: Clemon.

Dobrovol'skij, D. (1997a). Idiome im mentalen Lexikon. Ziele und Methoden der kognitivbasierten Phraseologieforschung. Trier: Wissenschaftlicher Verlag Trier.

Dobrovol'skij, D. (1997b). Idiome der lebendigen Sprache. Deutsch - Russisches Wörterbuch. Moskva. 
Dobrovol'skij, D. (1995). Kognitive Aspekte der Idiom-Semantik - Studien zum Thesaurus deutscher Idiome. Tübingen: Narr Verlag.

Donalies, E. (2009). Basiswissen Deutsche Phraseologie. Tübingen: Narr Francke Attempto Verlag.

Duden Das große Wörterbuch der deutschen Sprache in zehn Bänden (1999). Hrsg. vom Wissenschaftlichen Rat der Dudenredaktion. 3., völlig neu bearbeitete und erweiterte Auflage Mannheim u.a.: Dudenverlag.

Durèo, P. (1994). Probleme der allgemeinen und kontrastiven Phraseologie. Am Beispiel Deutsch und Slovakisch. Heidelberg: Gross.

Durèo, P. (2001). „Bekanntheit, Häufigkeit und lexikografische Erfassung von Sprichwörtern. Zu parömiologischen Minima für DaF“. In H. Buhofer et al. (Hrsg.) Phraseologiae Amor. Aspekte europäischer Phraseologie. Baltmannweiler: Schneider Verlag, 99-106.

Ehrhard, A.-F. (1999). „Zur Motiviertheit der Neg-Komponente in negativen Phrasemen: Negierung oder Negation». In F. Bravo et al. (Hrsg.). Phraseme und typisierte Rede. Tübingen: Niemeyer (Eurogermanistik 14), 39-50.

Elspaß, S.(1998). Phraseologie in der politischen Rede - Untersuchungen zur Verwendung von Phraseologismen, phraseologischen Modifikationen und Verstößen gegen die phraseologische Norm in ausgewählten Bundestagsdebatten. Wiesbaden: Opladen.

Ephras-Projekt (2006). Ein mehrsprachiges phraseologisches Wortmaterial. Ljubljana: Rokus.

Fleischer, Wolfgang (1982). Phraseologie der deutschen Gegenwartssprache. Leipzig: Niemeyer Max.

Fleischer, W. (1997). Phraseologie der deutschen Gegenwartssprache. Tübingen: Niemeyer, 2. Auflage.

Földes, C. and Wirrer, J.(Hrsg.) (2004). Phraseologismen als Gegenstand sprach- und kulturwissenschaftlicher Forschung. Baltmannsweiler: Schneider Verlag Hohengehren.

Funk, H. (2004). „Qualitätsmerkmale von Lehrwerken prüfen - ein Verfahrensvorschlag«. In Babylonia, 3: 41-47. Erreichbar unter: www.babylonia-ti.ch (28.12.2010).

(GER) Gemeinsamer europäischer Referenzrahmen für Sprachen: lernen, lehren, beurteilen (2001). Council of Europe. Council for Cultural Co-operation. Education Committee. Modern Languages Division. Berlin: Langenscheidt.

Glaboniat, M. et al. (2005). Profile deutsch. Berlin: Langenscheidt.

Götz et al. (Hrsg.) (1997). Langenscheidts Großwörterbuch Deutsch als Fremdsprache. Langenscheidt Verlag.

Gréciano, G. (1997). „Zur Festigkeit von Phraseologie. Eine Merkmalanalyse». In I. Barz and M. Schröder, (Hrsg.). Nominationsforschung im Deutschen. Festschrift für Wolfgang Fleischer zum 75. Geburtstag. Frankfurt u.a.: Peter Lang, 167-175.

Griesbach, H. and Schulz, D. (2000). 1000 deutsche Redensarten. Mit Erklärungen und Anwendungsbeispielen. Berlin etc.: Langenscheidt

Griesbach, H. and Ulrich, G. (1993). Mit anderen Worten. Deutsche Idiomatik, Redensarten und Redeweisen. München: Iudicum.

Hallsteinsdóttir, E., Šajánková M. and Quasthoff, U. (2006). »Phraseologisches Optimum für Deutsch als Fremdsprache. Ein Vorschlag auf der Basis von Frequenz- und Geläufigkeitsuntersuchungen«. In Linguistik online, 2:1-20.

Herzog, A. (1993). Deutsche Redewendungen von A-Z. Ein Übungsbuch für Anfänger und Fortgeschrittene. Leipzig: Baltmannsweiler, Schneider Verlag.

Hessky, R. (1997). „Feste Wendungen - ein heißes Eisen? Einige phraseodidaktische Überlegungen für den DaF». In DaF, 3:139-143. 
Redensarten-Index. Wörterbuch für Redensarten, Redewendungen, idiomatische Ausdrücke, feste Wortverbindungen. Erreichbar unter: http://www.redensarten-index.de/suche.php (5.1.2011).

Helbig, G. (2006). „Funktionsverbgefüge - Kollokationen - Phraseologismen. Anmerkungen zu ihrer Abgrenzung im Lichte der gegenwärtigen Forschung». In U. Breuer, and I. Hyverinen (Hrsg.). Wörter - Verbindungen. Festschrift für Jarmo Korhonen zum 60. Geburtstag. Frankfurt et al.: Peter Lang, 165-174.

Hessky, R. and Ettinger, S. (1997). Deutsche Redewendungen. Ein Wörter-und Übungsbuch für Fortgeschrittene. Tübingen: Niemeyer.

Jesenšek, V. (2008). Begegnungen zwischen Sprachen und Kulturen Beiträge zur Phraseologie. Bielsko-Biala: Akademia Techniczno-Humanistyczna.

Kempcke, G. et al. (2000). Wörterbuch Deutsch als Fremdsprache. Berlin, New York: de Gruyter Verlag.

Kispal, T. (1999). „Zur Behandlung von Sprichwörtern im DaF-Unterricht». In Deutsch als Fremdsprache, 36, 3: 166-169.

Kühn, P. (1994). „Pragmatische Phraseologie: Konsequenzen für die Phraseologie und Phraseodidaktik». In B. Sandig (Hrsg.). Tendenzen der Phraseologieforschung. Bochum: Brockmeyer.

Lemnitzer, L. (1997). Akquisition komplexer Lexeme aus Textkorpora. Tübingen: Niemeyer (Reihe Germanistische Linguistik 180).

Lorenz-Bourjot, M. and Lüger, H.-H. (Hrsg.) (2001). Phraseologie und Phraseodidaktik. Wien: Edition Praesens.

Lüger, H.-H. (1997). Anregungen zur Phraseodidaktik. In Beiträge zur Fremdsprachenvermittlung, 32: $69-120$.

Lüger, H.-H. (1999). Satzwertige Phraseologismen - Eine pragmalinguistische Untersuchung. Wien: Ed. Praesens.

Matulina, •. and Ćolarić, Z. (2007). „Phraseologismen in neueren kroatischen und bosnischen Lehrwerken des Faches DaF». In V. Jesenšek and M. Fabèiè (Hrsg.). Phraseologie kontrastiv und didaktisch. Neue Ansätze in der Fremdsprachenvermittlung. Maribor: Zora 47: 95-110.

Palm, C. (1997). Phraseologie - Eine Einführung. Tübingen: Narr. 2., durchgesehene Auflage.

Stein, S. (1995). Formelhafte Sprache - Untersuchungen zu ihren pragmatischen und kognitiven Funktionen im gegenwärtigen Deutsch. Frankfurt a. M.: Peter Lang

Valentin, P. (1999). „Unterm Phrasem das Zeichen». In B. Fernandez et al. (Hrsg.) Phraseme und typisierte Rede. Tübingen: Niemeyer (Eurogermanistik 14), 15-23.

Wotjak, B. (1999). „Zu textuellen Vernetzungen von Phraseologismen am Beispiel von Sprachwitzen». In B. Fernandez et al. (Hrsg.). Phraseme und typisierte Rede. Tübingen: Niemeyer (Eurogermanistik 14), 51-62.

Wotjak, B. (2001). «Phraseologismen im neuen Lernerwörterbuch - Aspekte der Phraseologiedarstellung im de Gruyter-Wörterbuch Deutsch als Fremdsprache». In B. Häcki et al. (Hrsg.) Phraseologiae Amor. Aspekte europäischer Phraseologie. Baltmannsweiler: Schneider Verlag Hohengehren (Phraseologie und Parömiologie 8), 263-279. 